\title{
Récits de mort et souvenir traumatique. Trames et traces lexicales des témoignages sur la Shoah
}

Death Narrative and Traumatic Memory: Narrative Spine and Lexical Traces in

Testimonies of Holocaust Survivors

Damon Mayaffre and Mahé Ben Hamed

\section{(2) OpenEdition}

Electronic version

URL: http://journals.openedition.org/aad/1836

DOI: $10.4000 /$ aad. 1836

ISSN: 1565-8961

\section{Publisher}

Université de Tel-Aviv

\section{Electronic reference}

Damon Mayaffre and Mahé Ben Hamed, « Récits de mort et souvenir traumatique. Trames et traces lexicales des témoignages sur la Shoah », Argumentation et Analyse du Discours [Online], 13 | 2014, Online since 14 October 2014, connection on 23 September 2019. URL : http:// journals.openedition.org/aad/1836 ; DOI : 10.4000/aad. 1836

This text was automatically generated on 23 September 2019

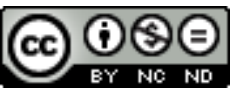

Argumentation \& analyse du discours est mis à disposition selon les termes de la licence Creative Commons Attribution - Pas d'Utilisation Commerciale - Pas de Modification 4.0 International. 


\section{Récits de mort et souvenir traumatique. Trames et traces lexicales des témoignages sur la Shoah}

Death Narrative and Traumatic Memory: Narrative Spine and Lexical Traces in Testimonies of Holocaust Survivors

Damon Mayaffre and Mahé Ben Hamed

\section{Introduction}

1 Les témoignages sur la Shoah sont depuis désormais plusieurs décennies, en France comme partout dans le monde, enjeux mémoriels pour la société et objet scientifique pour l'Académie. Parce que la Solution finale fut l'anéantissement programmé par les cendres d'un peuple, d'une religion, d'une mémoire, son échec durable, au-delà de la libération des camps, passe par le souvenir d'abord préservé, ensuite étudié de ce qui a été : la mémoire de la Shoah en étant un impératif éthique et politique est devenue un objet d'étude pour les sciences humaines et sociales.

Les récits de vie - que l'on appellera ici récits de mort - constitués de la narration des survivants du génocide prennent une forme plurielle et quasi incommensurable aujourd'hui dans les différents mémoriaux planétaires, mais constituent un corpus homogène quant à la volonté de "témoigner " ${ }^{1}$. Dans ce témoignage universel, nous avons prélevé 16 longs récits autobiographiques de déportés à Auschwitz pour la grande majorité, écrits a posteriori la plupart dans les années 2000, pour les soumettre à une étude textuelle instrumentée par l'informatique et la statistique (pour une description précise du corpus issu de la collection Témoignages sur la Shoah de la Fondation pour la Mémoire de la Shoah, cf. annexe 1). 
3 La problématique qui soutient le propos s'inscrit dans le programme international MATRICE (http://matricememory.fr) sur l'articulation entre mémoire individuelle et mémoire sociale, souvenirs personnels et représentation collective, narration individuelle et grand récit (Peschanski 2013). Nous posons que la mémoire - qui plus est, la mémoire d'un événement aussi majeur que la Shoah - est toujours sédimentation mémorielle, dans laquelle s'accumulent et se combinent souvenirs intimes du témoin et narration historique ou collective ; dans une dialectique qui demande à être démontée, matériau mnésique primaire et matériau historique secondaire s'articulent, jusqu'à l'amalgame, pour former un récit cohérent, en l'occurrence dix, 20 ou 60 ans après le drame.

4 Méthodologiquement, nous émettons l'hypothèse que les récits portent les traces lexicales du trauma initial tel qu'il a été encodé par la mémoire, comme ils portent les marques de la reconstruction narrative a posteriori. Aussi doivent-ils être soumis à un traitement qui aura pour objectif de sonder cette sédimentation lexicale et mémorielle complexe. Notamment, l'analyse de la trame co-occurrentielle et des réseaux lexicaux qui traversent et structurent le texte est susceptible de témoigner, entre mémoire et histoire, de la restitution-reconstruction problématique du souvenir traumatique.

\section{Cadres épistémologiques}

\subsection{Objet : récits de mort et souvenir traumatique}

5 Les témoignages sur la Shoah sont des récits de morts. Nous entendons par cette expression faire référence aux récits de vie tels que la sociologie, l'histoire ou la psychologie les ont longuement décrits et théorisés depuis presque un siècle ${ }^{2}$, mais en soulignant l'essence nécessairement tragique des manuscrits considérés. Le récit de mort renvoie à des événements hautement traumatiques (arrestation, déportation, asservissement, extermination des proches). En ce sens, le témoin mobilise une mémoire traumatisée telle que Cyrulnik (2012a et 2012b) a pu la décrire en détail. Dès lors, il ne s'agit pas seulement pour la victime de se souvenir, c'est-à-dire convoquer une mémoire enfouie, il ne s'agit pas encore seulement de travailler une mémoire que l'on admet remodelée par le temps, mais il s'agit aussi de restaurer une mémoire abîmée dans son encodage même pour en faire une mémoire vive, active, dicible et possiblement curative. Plus qu'une autre, la mémoire traumatique, pour être intégrée par les victimes, est remaniement, reconstruction, réélaboration à la faveur de la narration; c'est cette reconstruction, ou mise en cohérence discursive, qui est traquée dans cette contribution.

Le témoignage du Dr Viktor Frankl, interné à Auschwitz, est en effet instructif et invite l'observateur à la prudence. Relatant une scène particulièrement horrible de l'évacuation d'un cadavre de son infirmerie improvisée, Frankl (1988: 34) précise à l'endroit de l'analyste les failles de la mémoire :

Je me trouvais à l'autre bout de la baraque. [...] je buvais avidement ma soupe lorsque mes yeux furent attirés vers l'extérieur. Le cadavre qu'on venait d'y transporter me fixait de ses yeux vitreux. Deux heures auparavant, j'avais parlé à cet homme. Et maintenant, je sirotais ma soupe, dans l'indifférence la plus totale! Si je n'avais pas été surpris par ce manque d'émotion d'un point de vue professionnel, je n'aurais gardé aucun souvenir de l'incident. 
7 Par-là, c'est l'horreur de la Shoah qui est non pas inaccessible - puisque Frankl et tant d'autres finissent par témoigner ${ }^{3}$ - mais altérée, codée : elle doit être retravaillée par des témoins que la maltraitance physique et psychologique avait rendus en partie insensibles, sur le moment, au pire. Cette apathie émotionnelle, dirimante à une bonne mémorisation, et qui prend la figure extrême et problématique du "musulman $»^{4}$, ne pose évidemment pas la question négationniste de la réalité de la Shoah mais spécifie le statut du témoin dans sa nécessaire distanciation à sa propre réalité, car Frankl (1988 : 35) généralise le propos :

L'apathie, l'insensibilité aux émotions et le sentiment que plus rien ne le touchait constituaient les symptômes [...] des réactions psychologiques du prisonnier. [...] L'indifférence, le principal symptôme... faisait partie du mécanisme d'autodéfense de chaque prisonnier.

8 De manière générale enfin, l'étude des témoignages révèle que ce sont les conditions même de la mémoire qui se trouvent affectées par les modalités des faits. Particulièrement, on admet dans une posture kantienne élémentaire que le temps et l'espace sont les conditions de la mémorisation ${ }^{5}$. Sauf à rester à l'état de flashs, les récits de vie comme les récits de mort mettent nécessairement en scène une temporalité, un déroulement, une chronologie, une trame temporelle sur laquelle s'inscrivent de manière cohérente les épisodes. Or ce rapport essentiel au temps est abîmé par l'événement traumatique concentrationnaire ${ }^{6}$. La plupart des témoignages insistent en effet sur l'éternel recommencement de journées sans perspective au sein des camps, sur une vie sans calendrier, sur l'angoissante durée d'une vie éphémère et prise en otage. Tout juste remarque-t-on dans les récits la scansion structurante des saisons, synonymes de conditions de vie rendues plus difficiles encore en hiver par le froid ou en été par la chaleur, ou la scansion, routinière, de la journée de travail avec le réveil, le repas, le couché. Mais la plupart des témoignages soulignent l'incertitude calendaire, l'ignorance de la période d'internement, la perte des repères temporels, un temps social d'une certaine manière aboli : cette chronologie altérée se doit alors d'être retravaillée pour faire récit ${ }^{7}$.

9 Et le rapport à l'espace souffre des mêmes handicaps. Ce sont des hommes et femmes sortis du monde qui sont appelés à témoigner d'un théâtre qui pour l'essentiel leur échappe sur le moment. Déportées dans des wagons sans fenêtre - les témoins français font ainsi plusieurs milliers de kilomètres à travers l'Europe sans connaissance de leur destination -, internées dans des camps sans autre horizon spatial que la ronde du travail forcé, les victimes sont déracinées, dépaysées, au sens strict déboussolées. Comme la chronologie, les lieux du drame appellent à être reconstitués.

La perte de l'état civil remplacé par un numéro, la disparition des proches et la séparation familiale, l'isolement au sein d'une Babel macabre, dans laquelle ni le yiddish ni l'hébreux ne pouvaient tout à fait servir d'esperanto, contribuent fondamentalement à cette déstabilisation spatio-temporelle et s'attaquent aux racines identitaires mêmes de la mémoire individuelle : le témoin pour se souvenir doit d'abord se reconstruire en sujet là où les camps l'avaient assujetti. Les témoignages apparaissent ainsi aussi pour les survivants comme une ré-appropriation de leur identité - Paul Ricœur parlerait alors d'« identité narrative » - et comme la mise à proximité d'expérience dont ils furent, de fait, distanciés 8 . 


\subsection{Traces discursives : entre mémoire individuelle et récit collectif}

11 C'est à une archéologie de la mémoire que l'on veut se livrer ici en étudiant les "traces» (ce terme aussi est emprunté à Ricœur) dans le récit et en pressentant l'articulation nécessaire entre souvenirs vécus et reconstruction mémorielle.

L'objectif n'est pas de jeter le soupçon sur la véracité des témoignages ou de restaurer une l'histoire objective de la shoah, largement établie par ailleurs, dont les témoignages s'écarteraient : ici les témoignages sur la Shoah nous semblent seulement prototypiques, pour les raisons indiquées précédemment, du travail nécessaire entre un matériau primaire (des «impressions-affections", dirait Ricœur) et son épanouissement cognitif complexe qui passe par une mise en récit ou une élaboration discursive: il n'existe pas pour nous de faux témoins ou de témoins illégitimes de l'extermination mais seulement des témoins dont la mémoire - comme toute mémoire - est sans cesse élaborée et réélaborée9.

13 D'Aristote à Ricœur, les auteurs s'accordent pour définir la mémoire comme une marque, un palimpseste, une re-présentation : le sceau (l'instrument même, c'est-à-dire l'événement lui-même) est à jamais disparu et laisse sur le témoin seulement une empreinte que l'activité cérébrale du sujet et la société (le grand récit, la doxa) réélaborent sans cesse.

On observera cependant que notre objet d'étude est uniquement langagier : matériau supposément d'origine ou matériau réélaboré, c'est d'un matériau seulement verbal dont nous disposons. Reprenant la phénoménologie discursive de l'événement historique de Guilhaumou (2006) nous insistons sur « la donation linguistique » ${ }^{10}$ de la trace mnésique : la mémoire - pour peu qu'elle soit ambitieuse et à partir du moment où elle veut être partagée - advient et nous est donnée le plus souvent dans sa forme linguistique : des mots, des récits, un discours. Se souvenir, c'est avant tout dire un souvenir, raconter un souvenir, sauf à le garder pour soi sous la forme du chaos du ressenti $^{11}$. Dans les termes de la psychologie, il s'agit d'une mémoire verbale ou « explicite », non d'une mémoire a-verbale ou « implicite » (Cyrulnick 2012-b : 134 et ss.). Lorsque Ricœur pose dans son introduction de Temps et Récit que toute narration implique mémoire (1983: 27), nous suggérons symétriquement que le souvenir implique narration, toutefois, répétons-le, lorsqu'il prétend s'élever au rang de mémoire aboutie socialement partagée. Au risque de réduire la complexité d'une mémoire qui peut être visuelle, olfactive, gustative, etc. et que la littérature décrit volontiers, dans un premier mouvement, comme une « image ", c'est donc, pour notre part, seulement au sein du langage, c'est-à-dire, concrètement, au cœur du corpus - le corpus textuel des témoignages écrits - que l'on cherchera à décrypter dans les souvenirs de la Shoah la part originelle et la part rétrospective, à partir de traces lexicales qui les matérialisent.

\subsection{Méthode : co-occurrences textuelles et associations sémantiques en mémoire}

Les développements les plus récents en matière de logométrie (mesure du discours) ou de textométrie (mesure du texte) établissent les discours et les textes comme des entités réticulaires dans lesquels les unités linguistiques s'organisent. Au-delà du sac de mots ou de l'urne originels du lexicométricien (Muller 1977, Lebart et Salem 1994), un 
corpus de textes peut être décrit par la statistique textuelle comme un ensemble de structures sous-jacentes et organisées, ou réseaux co-occurrenttiels privilégiés (Brunet 2012-a). Et en passant de l'occurrence (l'unité seule) à la co-occurrence (l'unité dans ses relations avec les autres unités), nous opérons un saut qualitatif décisif qui nous mène du mot au thème, du thème au texte et du texte au sens.

Nous postulons que ces réseaux ou organisations co-occurrenttielles actualisent les associations en mémoire que les sciences cognitives tentent aujourd'hui de modéliser (par ex. Brunel et Lavigne 2009). Plus que jamais ici le récit lui-même est perçu avec (Ricoeur 1986: 156 et ss.) comme une «trace »ou recueil de «traces» d'une activité cérébrale et mémorielle. De manière pour l'heure simplement analogique, nous envisageons la structuration des réseaux co-occurrenttiels comme trace d'associations sémantiques préférentielles dans la mémoire du témoin, au sens de relations à la fois signifiantes (du point de vue linguistique/textuel) et significatives (du point de vue cognitif pour celui qui écrit). Et dans cette logique, autant que le lexique lui-même, ce sont ces relations ou associations, repérables statistiquement, qui portent pour nous le sens dans le texte et en mémoire.

Ces relations peuvent être formalisées et consignées dans une matrice mots $\mathrm{x}$ mots, représentation mathématique élémentaire que l'on constitue à partir du croisement des 300 substantifs les plus fréquents du corpus.

\begin{tabular}{|l|l|l|l|l|l|l|l|l|}
\hline & Affaire & Allemand & Année & Auschwitz & Baraque & Baraquement & Barbelé & Etc. \\
\hline Affaire & & $X$ & $Y$ & $Z$ & $\ldots$ & $\ldots$ & $\ldots$ & $\ldots$ \\
\hline Allemand & $X$ & & $\ldots$ & $\ldots$ & $\ldots$ & $\ldots$ & $\ldots$ & $\ldots$ \\
\hline Année & $Y$ & $\ldots$ & & $\ldots$ & $\ldots$ & $\ldots$ & $\ldots$ & $\ldots$ \\
\hline Auschwitz & $Z$ & $\ldots$ & $\ldots$ & & $\ldots$ & $\ldots$ & $\ldots$ & $\ldots$ \\
\hline Baraque & $\ldots$ & $\ldots$ & $\ldots$ & $\ldots$ & $\ldots$ & $\ldots$ & $\ldots$ & $\ldots$ \\
\hline Baraquement & $\ldots$ & $\ldots$ & $\ldots$ & $\ldots$ & $\ldots$ & & $\ldots$ & $\ldots$ \\
\hline Barbelé & $\ldots$ & $\ldots$ & $\ldots$ & $\ldots$ & $\ldots$ & $\ldots$ & & $\ldots$ \\
\hline Etc. & $\ldots$ & $\ldots$ & $\ldots$ & $\ldots$ & $\ldots$ & $\ldots$ & $\ldots$ & \\
\hline
\end{tabular}

18 A l'intersection d'une ligne et d'une colonne (i.e. dans les cellules du tableau) est recensé le nombre de fois $(\mathrm{X}, \mathrm{Y}, \mathrm{Z}$...) où le mot-ligne et le mot-colonne sont coprésents dans un même paragraphe. Ces intersections sont ainsi les mailles tressées des filslignes et des fils-colonnes, et constituent les composants sémantiques à partir desquels le tissu ou le tissage du texte - dont c'est d'ailleurs l'étymologie - émerge.

19 Cette matrice, parce qu'elle représente simplement mathématiquement le texte et le maillage sémantique du corpus, est le point de départ de nombreuses approches en Analyse de Données textuelles (ADT). Dans la suite de cette contribution, nous dissèquerons ce texte-matrice au travers divers traitements statistiques, en faisant 
dialoguer le niveau global (représentation vectorielle planaire, hiérarchique en classes ou encore en réseaux) et le niveau local (zooms sur certaines structurations discursives significatives ou sous-systèmes co-occurrenttiels particuliers).

\section{Résultats empiriques}

\subsection{Organisation globale du vocabulaire}

Un texte - ici les récits - peut donc être perçu comme un espace lexical organisé - ou espace réticulaire. Au niveau le plus général, cette organisation est appelé par les littéraires «textualité » (i.e. ce qui fait qu'un texte est un texte et non un chaos de mots); à un niveau inférieur, cette organisation composée de sous-systèmes peut définir des thèmes.

21 A la suite de Viprey (1997), il est d'abord possible de traiter la matrice de cooccurrences (tableau 1) par l'Analyse factorielle des Correspondances (Benzécri 1982). Les profils co-occurrenttiels des mots, consignés dans chaque ligne-colonne, sont ramenés à des vecteurs, dont on rend compte sur le plan par approximation mathématique.

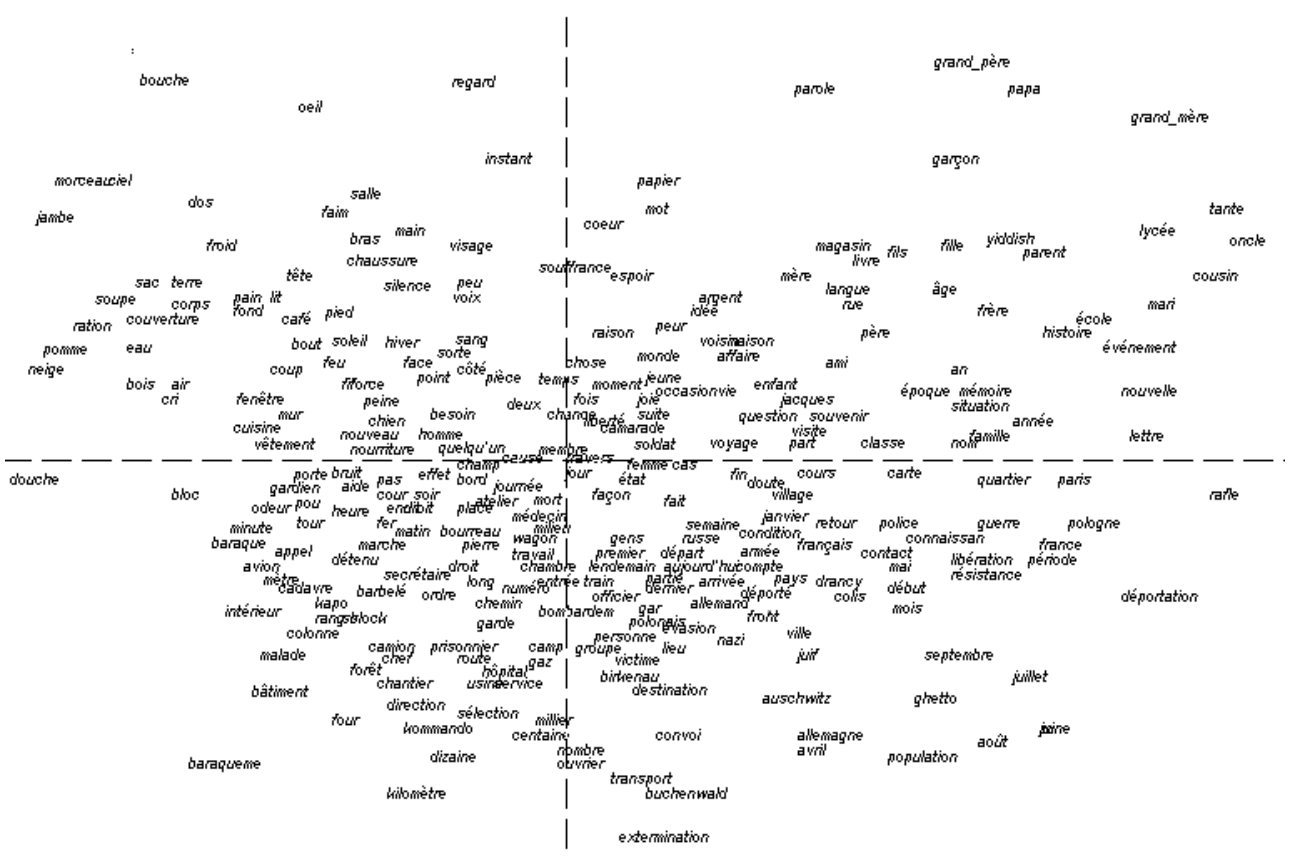

Figure 1 : Analyse factorielle des correspondances de la matrice mots $\mathrm{x}$ mots (sortie Hyperbase 9.0-2014)

Au-delà des thèmes sur lesquels nous reviendrons qui transparaissent en filigrane du graphique, nous émettons ici l'hypothèse que l'AFC distribue le vocabulaire, de gauche à droite, selon un axe «ressenti primaire" (à gauche) $\Leftrightarrow$ «reconstruction mémorielle » (à droite).

Il y aurait ainsi dans les récits de mort des survivants deux lexiques fondamentaux, sans doute indispensables l'un à l'autre et évidemment inscrits dans un continuum, mais distincts : un lexique servant à rendre compte de l'événement vécu tel qu'il fut encodé par la mémoire au moment des faits, c'est-à-dire consubstantiel ou contemporain au trauma initial; l'autre lexique, plus élaboré voire rétrospectif, 
permettant de mettre en scène le récit mémoriel, de le re-présenter, de lui donner sens social et acception historique.

\section{- A droite le vocabulaire reconstruit}

Par exemple, à l'extrême droite du graphique, les termes « rafle » et "déportation » n'appartiennent pas nécessairement au vocabulaire du déporté au moment des faits. Certes, ces mots existent avant la guerre, et il n'est aucunement exclu que certains contemporains aient pu commencer à les utiliser dans les camps. Mais ils restent rares alors, et ne s'imposent qu'au fil du temps à la faveur du récit collectif d'après-guerre. Le témoignage du corpus de référence du français moderne de Google Books, composé de quelque 43 milliards de mots, intégré aujourd'hui à Hyperbase (Brunet 2012b), montre qu'il s'agit en tout cas d'un vocabulaire que l'on qualifiera de " consolidé $~^{12}$ au cours des décennies post-Shoah.

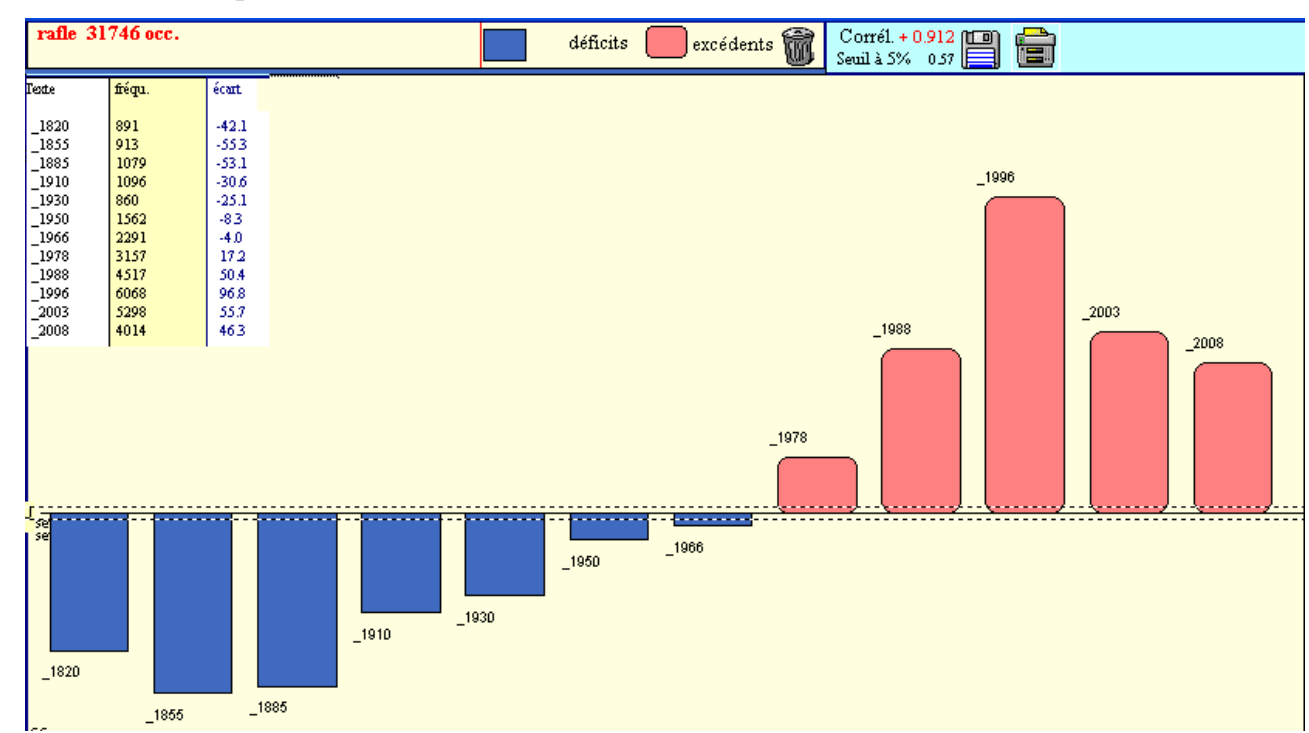

Figure 2 : Distribution de« Rafle » dans Google Books (1820-2008) (sortie Hyperbase 9.0-2014)

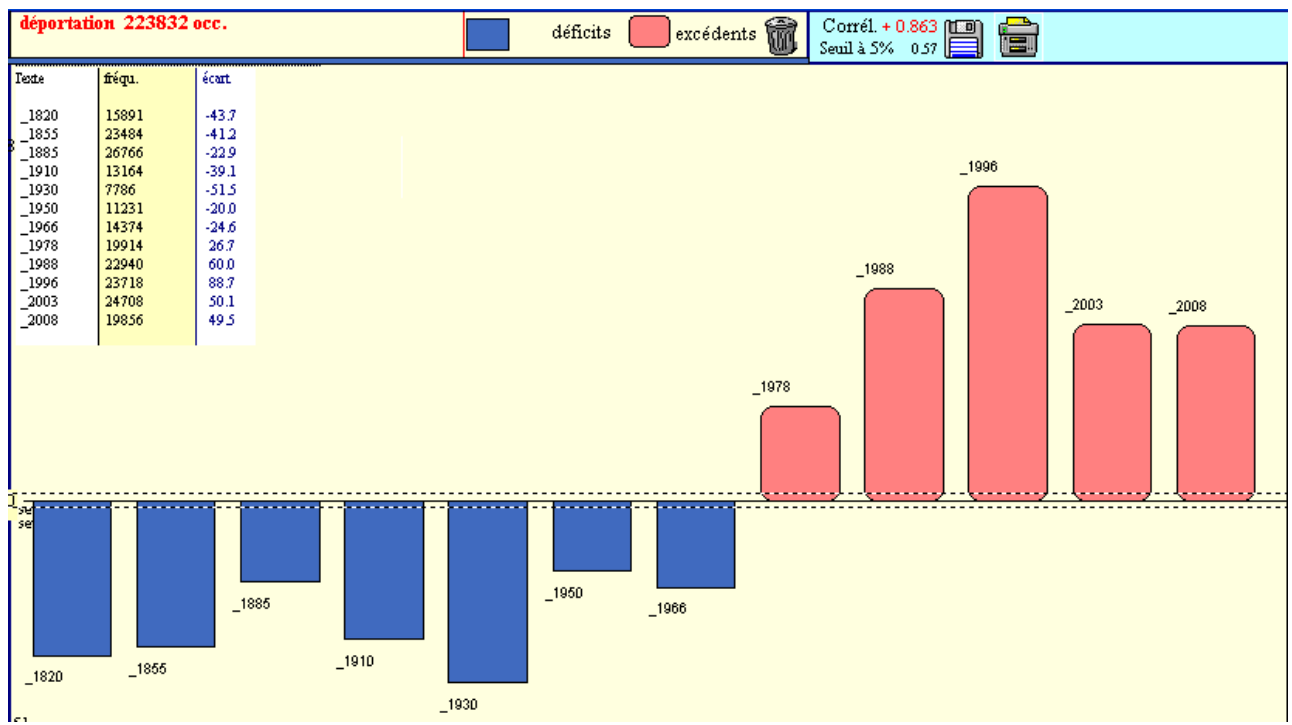

Figure 3 : Distribution de« déportation » dans Google Books (1820-2008) (sortie Hyperbase 9.0)

D'une certaine manière, à en croire Google Books, il faut attendre les années 1980 puis 2000, précisément au moment où sont écrits, traduits ou publiés la plupart de nos 
témoignages, pour voir ces mots être consacrés statistiquement par les historiens et la société entière ; et il n'est pas étonnant de voir les témoins (ou exceptionnellement leurs traducteurs) s'y conformer au moment de l'écriture de leur mémoire autour du début du 21e siècle.

La démonstration serait sans doute plus évidente avec l'utilisation du terme « Shoah » (présent dans le corpus mais qui n'apparait pas sur l'AFC faute d'un nombre d'occurrences suffisant). Son origine et son emploi sont bien sûr très anciens, mais sa pertinence pour qualifier le judéocide nazi est, on le sait, postdatée par rapport à la guerre. Du reste, la convocation des passages où le mot apparaît dans les témoignages du corpus montre que le terme relève moins de la mémorisation de l'événement que de la remémorisation ou même de la co-mémoration ${ }^{13}$ :

J'ai décidé d'entreprendre l'écriture de ce douloureux itinéraire que fut celui de ma famille et aussi le mien durant la Shoah. Ce sera sans doute un témoignage de plus écrit par un rescapé des camps d'extermination. Cependant, convaincue par ma famille et mes amis surtout, et sans doute aussi par l'âge, je ne voudrais pas achever le voyage de ma vie et le sursis qui m'a été accordé sans, à ma façon, rendre hommage à mes parents et aux six millions de Juifs victimes de la solution finale. [...] Nous en sommes à ce stade, soixante-quatre ans après l'amputation de notre famille par l'assassinat de mes parents et de ma grand-mère, exterminés dans les chambres à gaz de Pologne ${ }^{14}$.

Et le témoin conclut de manière explicite: «Le désespoir nous aurait saisis si nous n'avions été, dès notre retour, des hommes et des femmes convaincus qu'allait s'ouvrir un avenir de paix et de démocratie, une fois que le monde aurait pris connaissance de ce qui reçut par la suite le nom de Shoah.» (Nous soulignons.)

Les termes "mémoire", "souvenir» ou «histoire», situés comme "rafle» ou " déportation » dans la partie droite du graphique, illustrent bien ce travail réflexif de la mémoire sur elle-même ou du témoin sur son œuvre testimoniale : le survivant se remémore, et c'est cette remémoration - et non l'objet du souvenir lui-même - qui devient le sujet du discours. Les témoignages sur la Shoah passent ainsi inévitablement, comme le dirait Ricœur (2000), de l'acte testimonial personnel à la célébration sociale des défunts à qui le corps social doit rendre justice. Loin d'imaginer que cette dimension commémorative n'appartient pas à (ou trahit) la mémoire de la Shoah, gageons au contraire qu'il s'agit d'une dimension nécessaire au souvenir de l'extermination, comme finalement à tout souvenir ; une condition nécessaire à son élaboration. Matériaux mnésiques secondaires, pourrait-on considérer, ces mots sont les supports indispensables aux témoignages pour qu'ils puissent advenir et se dire aujourd'hui.

Dans ce cadre interprétatif du graphique, il devient facile de plaider que l'ensemble du vocabulaire familial (« oncle ", «famille », « grand-père », «frère ", «papa », etc.), à droite, prend ce caractère commémoratif, intime au témoin certes, mais à distance de l'événement originel. Hommage aux victimes disparues, célébration de la descendance : la famille est un nœud omniprésent des souvenirs de la Shoah tels qu'ils s'élaborent dans les récits du corpus, tout en étant, pour les raisons tragiques que l'on sait, quasi absente de la vie quotidienne des internés d'Auschwitz. «L'oncle » est toujours l'oncle disparu; le "frère » celui dont on a été séparé. Si mémoire familiale, vive et cruelle il y a, elle n'appartient qu'indirectement à la vie même des camps à l'exception des "mères" qui partagent l'arrestation, la déportation et les premiers temps de l'internement ${ }^{15}$. De manière plus évidente, et plus heureuse, la «fille», le «fils» ou le 
«mari» n'interviennent dans la vie du déporté qu'a posteriori. Le récit de mort y consacre pourtant une très grande place comme catharsis à l'extermination. Le souvenir traumatique - comme tout souvenir - compose ainsi autant avec le présent qu'avec le passé ; peut-être même le futur ${ }^{16}$.

\section{- A gauche le vocabulaire contemporain}

Si la partie droite du graphique organise ainsi un vocabulaire "postposé » ou rétrospectif à Auschwitz, la partie gauche doit être interprétée - par principe mathématique - comme son opposé : elle sélectionne un vocabulaire plus contemporain aux Lager, en lien direct avec l'événement tel qu'il fut subi. De fait, les termes sont plus concrets, précis, sensitifs, incarnés: "soupe ", "ration», "pomme de terre ", "jambe», "dos», «cadavre», " odeur», «couverture», etc. Les mots ici se rapprochent, semble-t-il, au plus près de l'image-souvenir ou de la sensation (olfactive, par exemple) sans que l'on puisse soupçonner une réélaboration importante.

De manière significative, l'espace ne s'expose plus dans les termes généraux des Etatsnations comme dans la partie droite du graphique ("Allemagne», "France», «Pologne»), mais se confine autour du «baraquement» ou des «baraques», du «bloc » ou « block », de la « cour » entourée de « barbelés », etc.

Les marqueurs de temps renvoient, comme pressenti plus haut, aux saisons climatiques ou à l'instant concret («l'hiver ", le «soleil », le «matin », la «minute ») là où le vocabulaire de droite faisait référence aux mois calendaires d'un temps social et officiel - sans doute inaccessible aux déportés (" juin », « juillet », « septembre », etc.).

De la même manière, si à droite du graphique, on trouvait avec «déportation" ou «rafle » déjà évoqués, les termes «juif » et "nazi » ou encore "résistance » et «libération » qui laissent comprendre une mise en cohérence historique de la Shoah autour de la judéité et de la guerre contre l'hitlérisme, la description de l'organisation des camps à gauche du graphique est plus élémentaire, sans considération politique ou religieuse, avec des « détenus », des « kapos », des « gardiens » ou des « bourreaux ».

Tableau 1 : Hypothèse interprétative de la distribution du vocabulaire selon le premier facteur :

\begin{tabular}{|l|l|l|}
\hline $\begin{array}{l}\text { Type de } \\
\text { vocabulaire }\end{array}$ & $\begin{array}{l}\text { RÉ-ÉLABORÉ } \\
\text { CO-MÉMORIEL, HISTORIQUE } \\
\text { (partie droite de l'axe horizontal) }\end{array}$ & $\begin{array}{l}\text { VÉCU } \\
\text { MÉMORIEL, PERSONNEL } \\
\text { (partie gauche de l'axe horizontal) }\end{array}$ \\
\hline SPATIAL & $\begin{array}{l}\text { Allemagne, Auschwitz, Drancy, } \\
\text { France, ghetto, pays, Pologne, etc. }\end{array}$ & $\begin{array}{l}\text { baraque, baraquement, barbelés, } \\
\text { bâtiment, bloc, block, chantier, four, etc. }\end{array}$ \\
\hline TEMPOREL & $\begin{array}{l}\text { janvier, juin, juillet, mois, septembre, } \\
\text { etc. }\end{array}$ & $\begin{array}{l}\text { hiver, matin, minute, soleil, etc. } \\
\text { SOCIO- } \\
\text { POLITIQUE }\end{array}$ \\
\hline $\begin{array}{l}\text { Déportation, juif, nazi, rafle, } \\
\text { résistance, libération, etc. }\end{array}$ & $\begin{array}{l}\text { Bourreaux, détenus, cadavres, gardes, } \\
\text { gardiens, kapos, prisonniers, etc. }\end{array}$ \\
\hline FAMILIAL & $\begin{array}{l}\text { Cousin, mari, grand-mère, grand- } \\
\text { père, oncle, tante, etc. }\end{array}$ & - \\
\hline
\end{tabular}




\begin{tabular}{|l|l|l|}
\hline SENSITIF & - & $\begin{array}{l}\text { Jambe, ration, soupe, eau, pain, corps, } \\
\text { froid, malade, etc. }\end{array}$ \\
\hline
\end{tabular}

Au final, il nous paraît artificiel d'opposer mémoire et rétrospection puisque toute mémoire, par définition, est rétrospective (convoquer maintenant un événement passé). L'interprétation que nous avons donnée de l'axe horizontal (gauche/droite) ne fonctionne ici qu'aux extrêmes, et il aurait été possible d'insister au contraire sur le continuum de l'axe et l'entrelacs lexical que constitue la partie centrale du graphique. Néanmoins, nous semblons distinguer, dans le tissage lexical des textes, des structures qui renvoient plus ou moins directement à l'événement ou participent au contraire à sa mise en récit historique.

\subsection{Thèmes structurants et classes de mots}

Le traitement de la matrice par l'analyse factorielle fait apparaître à grand trait une organisation fondamentale du vocabulaire, et un regard plus localisé permettrait sans doute de mettre à jour, empiriquement, quelques «isotropies» (groupements distributionnels) du discours (Viprey 1997). Cependant, à ce stade, aucune classification $\mathrm{du}$ vocabulaire n'a été opérée, rendant incertaine l'identification de thèmes dans le récit.

En appliquant la méthode de Classification descendante hiérarchique (CDH) « Alceste » (Reinert 1993) via le logiciel libre «Iramuteq $~^{17}$, sur le corpus des témoignages ramené à son vocabulaire fondamental (i. e. les 300 substantifs qui nous occupent) ${ }^{18}$, il est possible d'objectiver et de hiérarchiser cinq «classes» dont l'interprétation converge avec nos premiers résultats.

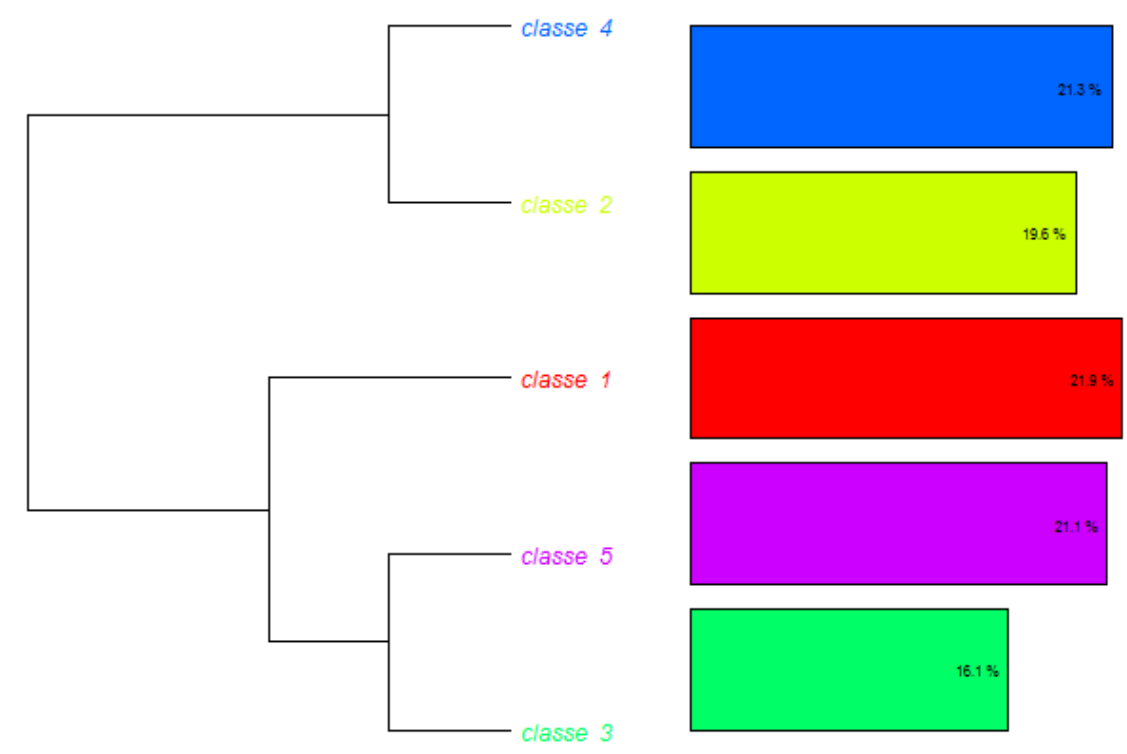

Figure 4 : Classification ascendante hiérarchique du vocabulaire du corpus (sortie Iramuteq)

Selon Reinert (1993), puis Marchand et Ratinaud (2012), ces classes peuvent être assimilées à juste titre à des thèmes, des «mondes" ou des tropes, au sens où elles 
mettent à jour des organisations d'ordre sémantique; et ici le fait de travailler seulement sur des substantifs favorise cette interprétation sémantico-thématique.

Le vocabulaire des cinq classes projeté sur le plan décline cinq ensembles thématiques remarquablement cohérents pour l'analyste (figure 5).

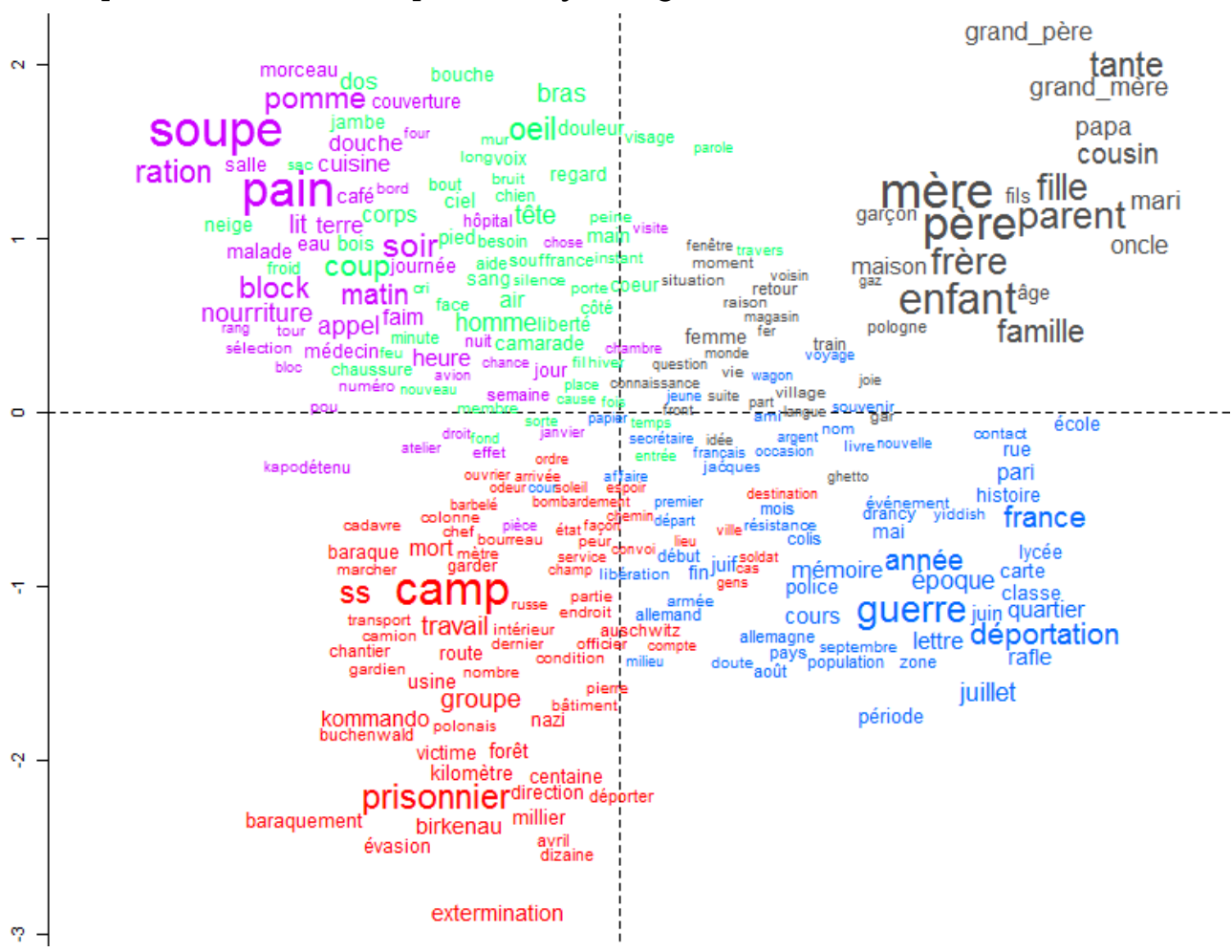

Figure 5 : Projection des cinq classes du corpus (sortie Iramuteq)

La partie droite concentre grosso modo et logiquement le même vocabulaire réélaboré que précédemment (cf. figure 1), mais avec l'identification de deux thèmes fondamentaux: le témoignage généalogique d'une part (en noir), le témoignage historique d'autre part (en bleu). Dans les deux cas, le vocabulaire transcende l'événement génocidaire stricto sensu c'est-à-dire le ici et maintenant des camps. Plus que jamais, il faut comprendre ces deux classes comme des soubassements nécessaires au récit sur la Shoah : pour témoigner, notamment dans les années 2000, le survivant s'appuie sur une histoire généalogique d'une part et sur l'histoire politique d'autre part. Ainsi de longs passages du corpus, notamment sur l'histoire familiale précédant ou succédant la guerre, pourraient paraître hors sujet ou éloignés du projet éditorial de la collection "Témoignage sur la Shoah ", mais leur récurrence statistique - pour ne pas dire leur présence systématique dans les livres du corpus - prouve leur nécessité dans le récit.

41 A gauche, trois thèmes ressortent, relevant tous trois d'un souvenir à proximité de l'événement. La classe 3 et la classe 5 (en vert et en violet), intimement liées dans la classification hiérarchique, organisent l'expression du souvenir dans sa dimension la plus quotidienne, avec, pour la classe 3, l'expression de la souffrance alimentaire autour de la « ration», des «morceaux » de " pain» ou de la «soupe » de la «pomme de terre », et pour la classe 5 , l'expression de la souffrance physique avec le « dos », les «bras ", les « jambes », le «sang », le « froid ». Loin d'une tonalité commémorative ou historiographique, les extraits contenant ce lexique attestent de leur dimension 
testimoniale intime ou, au sens fort, littéraire et étymologique, leur dimension anecdotique.

Le soir, pendant l'appel, on nous donnait notre ration solide : un morceau de pain carré, noir, compact, un peu gluant, accompagné d'une plaquette de margarine ou d'un morceau de saucisson de foie, ou d'une cuiller de confiture; de temps en temps, nous recevions aussi dans la main une poignée de betteraves cuites ${ }^{19}$.

La classe 1 enfin, la plus nombreuse, située elle aussi à gauche du graphique, et rattachée aux deux précédentes sur l'CHD, organise le souvenir du camp dans son organisation matérielle, sociale et carcérale. Constituée par les mots "camp », "prisonnier", "mort», elle permet au témoin de décrire concrètement le fonctionnement industriel d'Auschwitz comme le " camp de la mort » ses " camions », ses « colonnes de prisonniers », ces « marches » etc.

Histoire (généalogique ou politique) et mémoire (intime ou concrète des camps) semblent ainsi pouvoir se distinguer à nouveau même si comme l'affirme Paul Ricœur, elles ne sauraient entrer en concurrence. Nous allons voir que c'est parfois au sein même des récits qu'elles s'intriquent, s'épaulent ou se combinent pour former témoignage.

\subsection{Réseaux : de l'organisation globale du vocabulaire aux constellations lexicales locales}

\subsubsection{Communautés lexicales}

Nous proposons une ultime vision statistique de la structuration lexicale des récits de mort, en convoquant les travaux récents sur les réseaux pour confirmer l'existence de plusieurs structures lexicales appelées "communautés", ou groupement lexicaux à forte connectivité, à l'intérieur des récits de mort du corpus.

La même matrice co-occurrenttielle mots x mots décrite ci-dessus (tableau 1) a été soumise au logiciel Gephi ${ }^{20}$ qui permet de dessiner un graphe de co-occurrences dans lequel chaque mot est relié aux autres en fonction de ses accointances cooccurrenttielles (Tauveron 2011) ${ }^{21}$. Dans ce graphe (figure 6) difficilement lisible à cause du nombre de nœuds (les mots) et de liens (relations co-occurrenttielles), les algorithmes de modularité issus des travaux de Newman et Girvan (2004), puis de Blondel et al. (2008) permettent d'objectiver des "communautés " ou sous-systèmes lexicaux à forte connectivité relativement au reste du réseau, selon deux logiques complémentaires: forte relation intra-communautaire (les mots d'une même communauté entretiennent de fortes relations co-occurrenttielles) et faible relation inter-communautaire (les mots de deux communautés différentes entretiennent de faibles relations de co-occurrences - figure 6). 


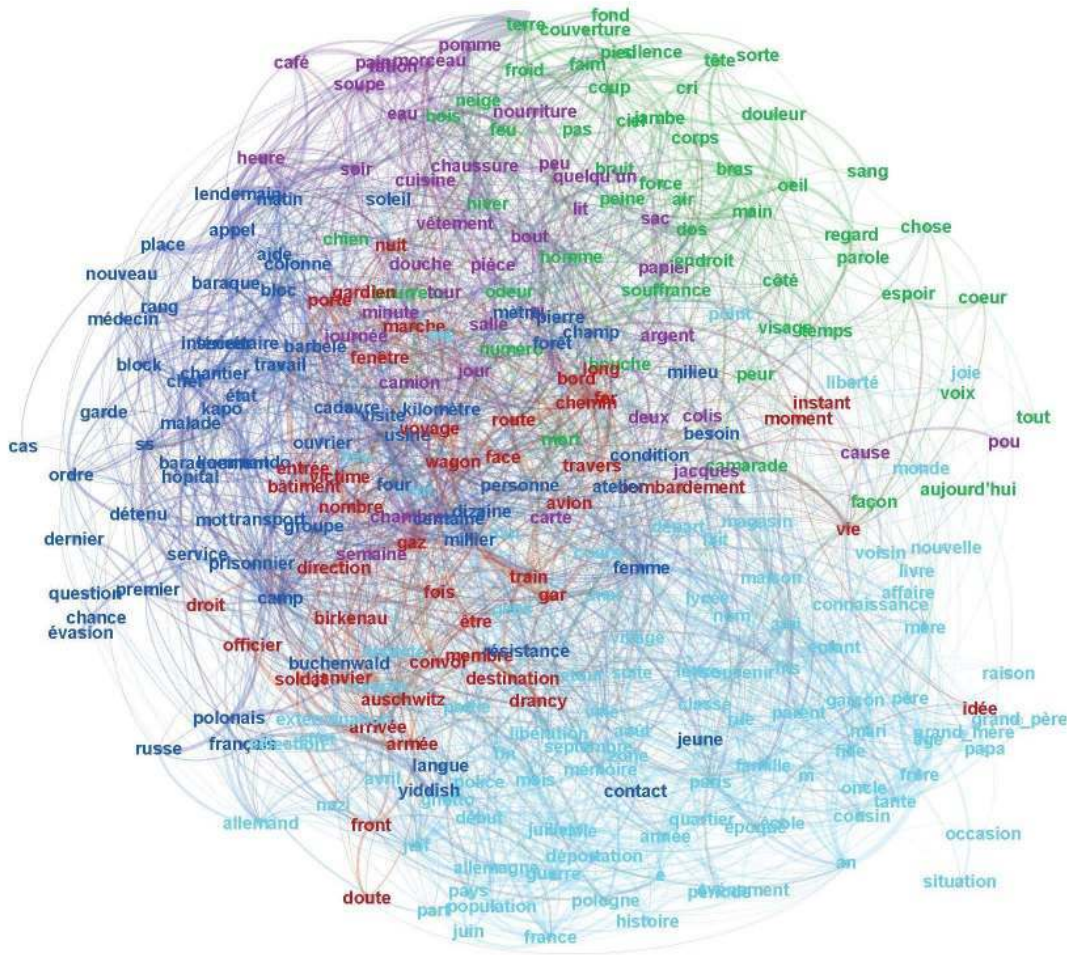

Figure 6 : Réseau des mots du corpus (sortie Gephi) porosité relative des lexiques. trame textuelle du corpus (figure 7).

Quoique d'essence mathématique différente de l'AFC (figure 1) ou de la CDH (figure 5), le réseau retrouve de manière concordante les résultats des études antérieures. Les cinq communautés ici calculées peuvent être rapprochées, malgré les nuances, aux classes d'Iramuteq qui elles-mêmes faisaient écho aux corrélats d'Hyperbase.

Mais au-delà de la confirmation des résultats et de certaines nuances qu'il conviendrait d'apporter ${ }^{22}$, la représentation du texte en réseaux de mots rend aussi compte de l'intrication des lexiques «mémoriels » et « historiques » dont on pouvait pressentir la non-étanchéité. $\mathrm{Si}$, du fait de notre questionnement, le risque existe de caricaturer la dichotomie lexicale à partir des sorties graphiques de l'AFC ou la $\mathrm{CDH}$, le réseau textuel nous met en demeure de rendre sa part à la continuité de la distribution lexicale, et à la

Particulièrement, le calcul de centralité (Betweenness centrality) permet de repérer les mots centraux versus les mots excentriques dans le réseau général, c'est-à-dire dans la

Les mots centraux sont bien sûr souvent les mots fréquents. Mais leur caractéristique définitionnelle est de multiplier leurs relations avec les autres mots, y compris, le cas échéant, avec ceux des communautés concurrentes.

Ainsi, par ordre hiérarchique, le terme «camp» est le plus central dans les textes 


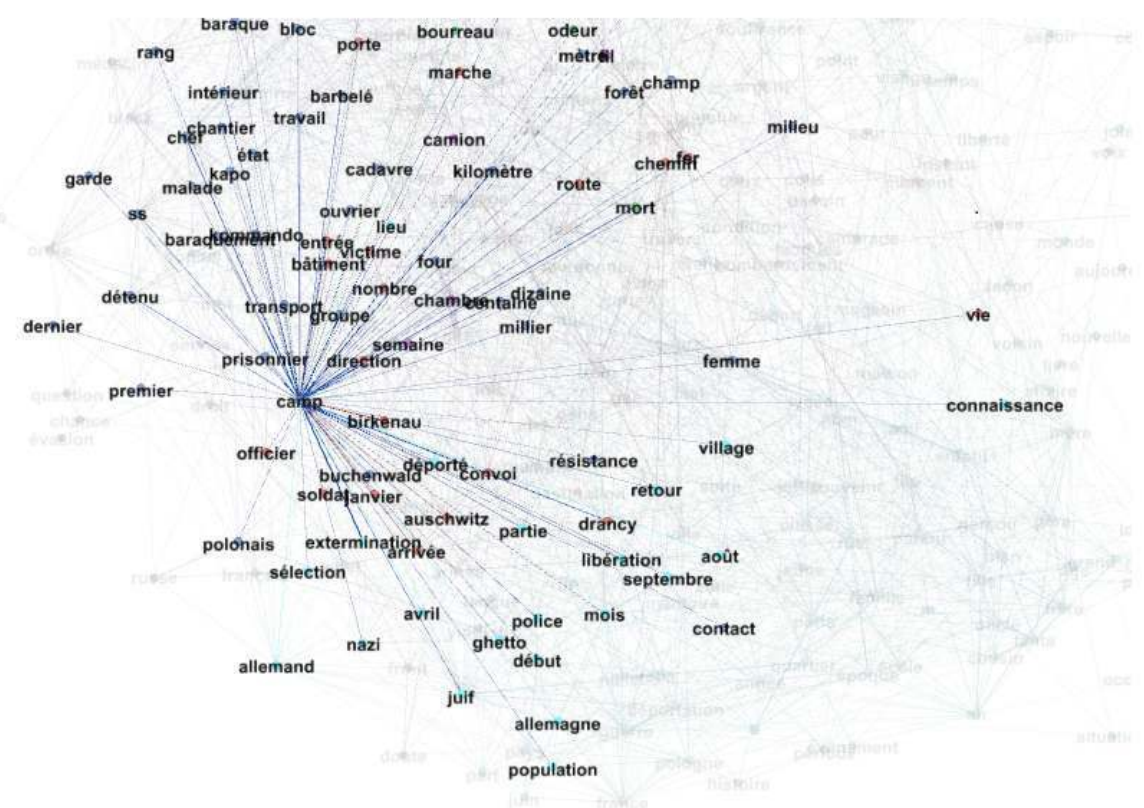

Figure 7 : Relations co-occurrenttielles de « camp » dans le réseau (sortie Gephi)

51 Nous pouvons supposer qu'il s'agit d'une brique lexicale essentielle des récits du corpus relevant sans doute à la fois de la mémoire et de l'histoire de l'extermination. Luimême rattaché à une communauté donnée (en bleu foncé, une communauté relevant de la description matérielle des camps), il entretient évidemment des liens avec les mots « détenu », « prisonnier ", « Kapo », etc. Cependant, il transcende sa communauté d'origine pour s'intriquer avec d'autres mots d'autres communautés comme, en bleu clair, un lexique plus historique («libération», "allemand», "population», « déporté »), ou en rouge avec « convoi », « crime », « vie ».

Plus intéressant, le terme " juif » apparaît aussi très bien situé dans le classement de la Betweenness centrality, avec une forte centralité dans le réseaux, confirmant mais nuançant le constat antérieur - il est à situer plutôt du côté du lexique historique que du côté du lexique mémoriel. 


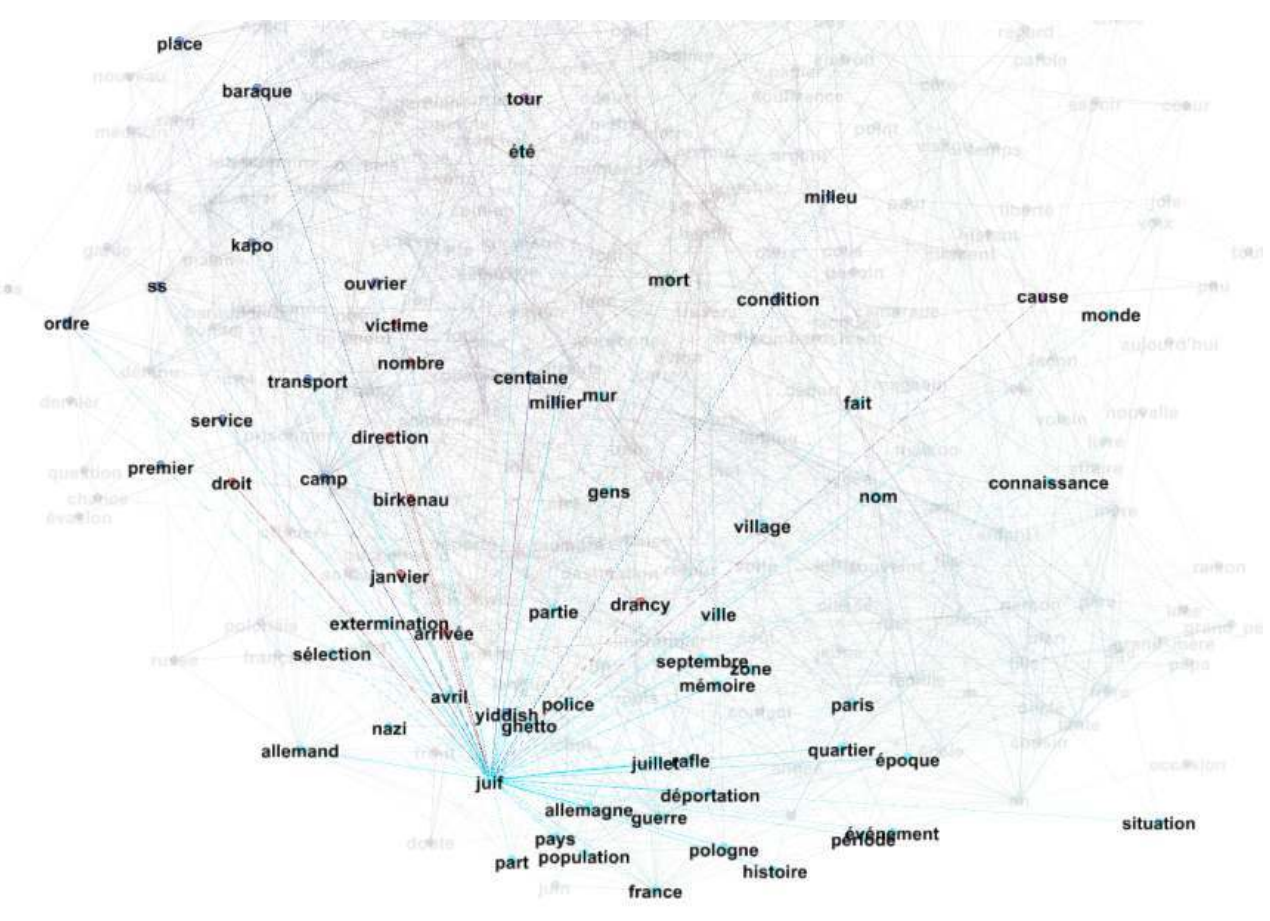

Figure 8: Relations co-occurrenttielles de «juif » dans le réseau (sortie Gephi)

En bleu clair, le mot appartient certes à la communauté composé d'un lexique sinon rétrospectif en tout cas réélaboré, avec " rafle », « histoire », " Pologne », " janvier ", «avril» etc. Mais le mot irradie le récit sur la Shoah au-delà, et on le retrouve à proximité des «baraques", du "camp», des "victimes", des «ouvriers», de la «mort». Central dans le récit, «juif» peut être considéré, avec d'autres («camp», «mort», etc.), comme une passerelle lexicale entre histoire et mémoire de la Shoah. Dans le feuilleté lexical du texte, il apparaît comme intermédiaire et constitue un pont et une permanence lexicale susceptible de rapprocher des passages relevant proprement du témoignage et ceux relevant de la mise en récit historique.

Beaucoup d'occurrences peuvent être ainsi extraites de passages historiques de ce genre : « En 1944, les Allemands exigèrent la déportation des JUIFS de Hongrie et depuis le printemps, des milliers et des milliers de JUIFS finissaient à Birkeneau ${ }^{23}$. - Mais certaines occurrences renvoient à la crue quotidienneté des camps :

Un dimanche, à midi, en passant devant une baraque, il aperçoit un JUIF en train de manger quelque chose. Il s'approche de lui, lui ordonne d'ouvrir la bouche et de montrer ce qu'il est en train de mâcher. Celui-ci mangeait un navet volé. De surprise, le prisonnier est littéralement cloué au sol, bouche grande ouverte. En un clin d'œil, Pröfi dégaine son pistolet et lui tire directement une balle dans la bouche ${ }^{24}$.

Cette centralité de "camp» ou de "juif» contraste dès lors avec la spécialisation d'autres mots dans le corpus qui ont un faible score de Betweenness centrality. Pour exemple, le terme "tante ", comme beaucoup de mots généalogiques, ne traverse que rarement sa communauté d'origine, pour entretenir des relations pratiquement uniquement avec les mots de son espèce. 


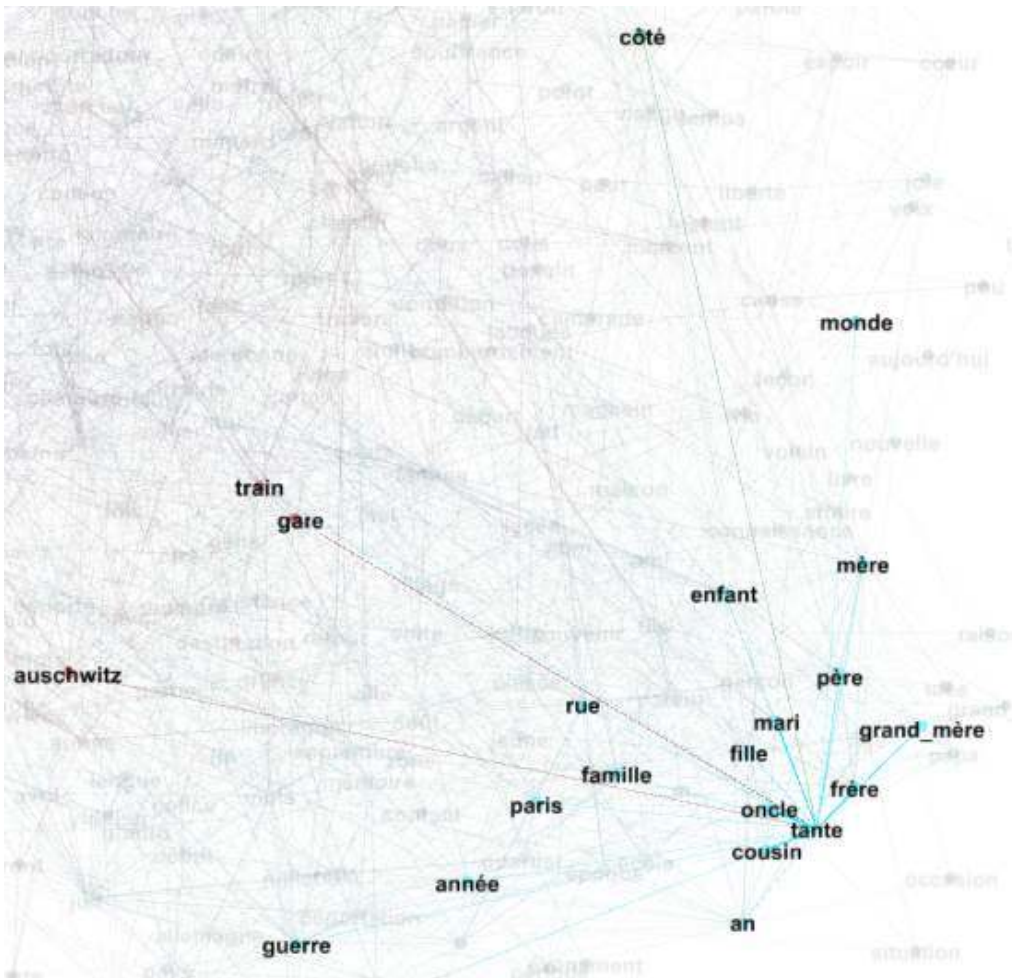

Figure 9 : Relations co-occurrentielles de « tante » dans le réseau (sortie Gephi)

"Extermination ", autre exemple, plus pertinent, apparaît lui-aussi comme un mot spécialisé dans les témoignages: il ne s'associe qu'avec peu de mots, et ceux-ci apparaissent très liés au grand récit.

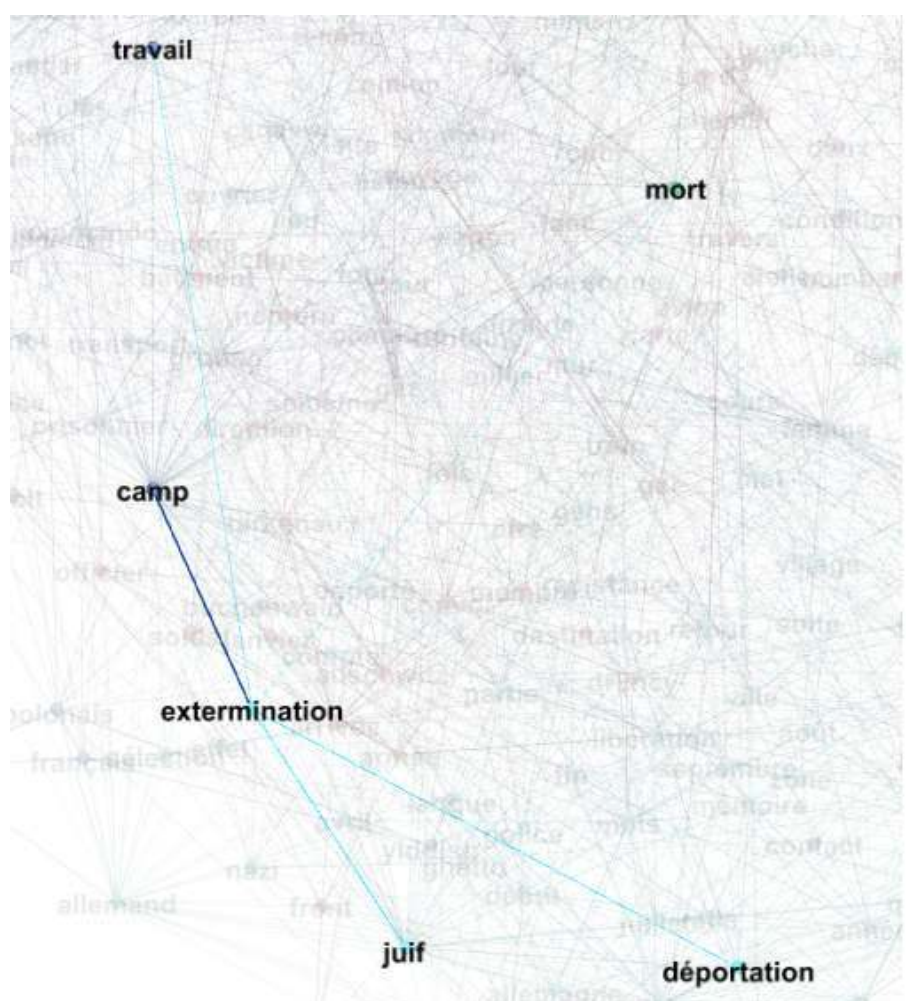

Figure 10. Relations co-occurrentielles d'« extermination » dans le réseau (sortie Gephi) 
Au risque d'être caricatural, citons par exemple ce passage relevant explicitement de la narration historique : «La France, après la Belgique, les Pays-Bas, le Luxembourg, était envahie par l'armée allemande. Hitler pouvait commencer la réalisation de son rêve nazi : la déportation et l'EXTERMINATION des Juifs des territoires occupés $»^{25} \mathrm{Ou}$ celuici, où le témoin établit un bilan tragique mais nécessairement a postériori :

Et dans ce cas précis, la méticulosité de la machine d'EXTERMINATION nazie frôlait la perfection. A peine une dizaine de prisonniers de Treblinka II ont survécu au camp d'extermination. Ce sont les prisonniers qui ont réussi à s'évader le 2 août $1943 \ldots{ }^{26}$.

Ainsi, à la dichotomie d'abord mise en jour entre lexique mémoriel et lexique commémoratif, il convient d'ajouter une seconde distinction entre lexique spécialisé dont il devient facile de déterminer l'origine et la fonction dans le récit, et un lexique central qui innerve le texte et apparie aussi bien la mémoire que l'histoire de la Shoah.

\section{Conclusion}

Ces 15 dernières années, sous l'impulsion des travaux de Ricœur, l'historiographie témoigne d'une grande effervescence à propos du couple notionnel mémoire et histoire. Et la commémoration du centenaire de la guerre de 1914 s'ouvre aujourd'hui, en France, notamment par la ré-interrogation de l'œuvre, connue depuis longtemps, de Jean Norton Cru autour du statut des témoins et du témoignage dans l'établissement des faits historiques.

Les travaux les plus récents essayent d'appréhender la dialectique entre mémoire personnelle et histoire collective moins en termes dichotomiques que complémentaires, et moins de manière statique que dynamique: l'histoire, aussi objectivante soit-elle, ne peut que difficilement ignorer les mémoires; la mémoire du sujet, aussi subjective et intime soit-elle, ne peut s'énoncer sans s'inscrire dans l'histoire ou le grand récit. Si la mémoire apparaît à Ricœur comme "matrice de l'histoire ", gageons à l'inverse que l'histoire - c'est-à-dire la mise en cohérence historique - apparaît comme le support nécessaire de la mémoire individuelle d'un événement majeur comme la guerre.

61 Cette contribution a essayé de montrer que dans la narration des survivants de la Shoah les méthodes d'ADT, autour des co-occurrences, peuvent mettre à jour, dans la complexité textuelle, un matériau lexical pouvant relever, aux deux pôles d'un continuum, des souvenirs les plus intimes et de la réélaboration historique.

D'un point de vue cognitif comme d'un point de vue textuel, il serait bien sûr vain d'imaginer un usage et une fonction uniques des mots : dans le texte comme dans le cerveau, les mots doivent pouvoir être mobilisés pour plusieurs finalités. Cependant, l'organisation lexicale du récit - c'est-à-dire la trame co-occurrentielle du corpus atteste, le cas échéant, une spécialisation variable, comme si l'amorçage (d'un point de vue cognitif) ou l'accroche (d'un point de vue textuel), par tel ou tel mot, devaient prédire, toutefois statistiquement, l'expression d'un souvenir primaire restitué ou d'un souvenir secondaire réélaboré. 


\section{BIBLIOGRAPHY}

\section{Méthodes ADT}

Ben Hamed, Mahé \& Damon Mayaffre. 2012. « Saisir le sens dans les deux sens : exploration de la portée interprétative de l'énergie et de la disponibilité ", JADT 2012, édité par A. Dister, D. Longrée, G. Purnelle (Bruxelles : Université de Liège / Facultés Universitaires Saint-Louis), 121-134

Benzécri, Jean-Paul. 1982. L'analyse des données (Paris : Dunod)

Blondel, Vincent \& al. 2008. « Fast unfolding of communities in large networks ». Journal of Statistical Mechanics: Theory and Experiment 10 (http://stacks.iop.org/1742-5468/2008/i=10/ $\mathrm{a}=\mathrm{P} 10008)$

Brunel, Nicolas \& Frédéric Lavigne. 2009. « Semantic Priming in a Cortical Network Model ». The Journal of Cognitive Neuroscience 21:12, 2300-2319

Brunet, Etienne. 2012a. « Nouveau traitement des cooccurrences dans Hyperbase », Corpus 11, 219-246

Brunet, Etienne. 2012b. « Au fond du Goofre. Un gisement de 44 milliards de mots », JADT, 8-20 Lebart, Ludovic \& André Salem. 1994. Statistique textuelle (Paris : Dunod)

Mayaffre, Damon. 2008. « De l'occurrence à l'isotopie. Les cooccurrences en lexicométrie », Sémantique \& Syntaxe $9,53-72$

Mayaffre, Damon. 2014. « Plaidoyer en faveur de l'Analyse de Données co(n)Textuelles Parcours cooccurrentiels dans le discours présidentiel français (1958-2014)», Emilie Néé, Jaen-Michel Daube, Mathieu Valette \& Serge Fleury (Éds). Actes des JADT 2014 (12es Journées internationales d'analyse statistiques des données textuelles) (Paris : Inalco-Sorbonne nouvelle), pp. 15-32 [en ligne : http://lexicometrica.univ-paris3.fr/jadt/jadt2014/01-ACTES/01-JADT2014.pdf, consulté le 10 octobre 2014]

Muller, Charles. 1977. Principes et Méthodes de statistique lexicale (Paris : Hachette)

Newman, Mark \& Michelle Girvan. 2004. « Finding and evaluating community structure in networks ». Physical Review E, 69 (026113)

Marchand, Pascal \& Pierre Ratinaud. 2012. Être Français aujourd'hui (Mayenne : Les Liens qui libèrent)

Reinert, Max. 1993. "Les mondes "lexicaux" et leur "logique" à travers l'analyse statistique d'un corpus de récits de cauchemars », Langage et société 66, 5-39

Tauveron, Matthias. 2012, « De la cooccurrence généralisée à la variation du sens lexical », Corpus $11,167-189$

Viprey, Jean-Marie. 1997. Dynamique du vocabulaire des Fleurs du mal. (Paris : Champion)

\section{Mémoire, histoire, Shoah}

Agamben, Giorgio. 1999. Ce qui reste d'Auschwitz : l'archive et le témoin (Paris : Payot).

Azouvi, François. 2012. Le mythe du grand silence. Auschwitz, les Français et la mémoire (Paris: Fayard) 
Cyrulnik, Boris. 2012a. Mémoire et traumatisme : l'individu et la fabrique des grands récits (entretien avec D. Peschanski) (Paris : INA)

Cyrulnik, Borris. 2012b. « Mémoire traumatique et résilience », D. Peschanski (dir.), Mémoire et mémorialisation, (Paris : Hermann), 133-143

Dosse, François. 1998. « Entre Histoire et mémoire. Une histoire sociale de la mémoire », Raison présente $128,5-24$

Frankl, Viktor. 1988. Découvrir un sens à sa vie avec la logothérapie (Paris : Éditions de l'Homme) Guilhaumou, Jacques. 2006. Discours et événement. L'histoire langagière des concepts (Besançon : PUFC)

Jablonka, Ivan \& Annette Wieviorka. 2013. Nouvelles perspectives sur la Shoah (Paris : PUF) Joutard, Philippe. 2013. Histoire et mémoires, conflits et alliance (Paris : La Découverte) Levy, Primo. 1988 (éd. française). Si c'est un homme (Paris : Pocket)

Levy, Daniel \& Natan Sznaider. 2006. The Holocaust and Memory in a Global Age (Philadelphia: Temple University Press)

Peschanski, Denis (éd.). 2013. Mémoire et mémorialisation - Volume 1 : De l'absence à la représentation (Paris : Hermann)

Rastier, François. 2005. Ulysse à Auschwitz : Primo Levi, le survivant (Paris : Cerf)

Ricoeur, Paul. 1983-1991. Temps et récit, t.1 L'intrigue et le récit historique, et t.3, Le temps raconté (Paris : Seuil)

Ricoeur, Paul. 2000 rééd.. La mémoire, l'histoire, l'oubli (Paris : Seuil)

Wieviorka, Annette. 2002. L'ère du témoin (Paris : Hachette)

\section{Annexe : le corpus}

Parus aux Éditions Le Manuscrit, FMS- Collection Témoignages de la Shoah :

Altmann, Erich. 2007. Face à la mort, Auschwitz-Buchenwald-Oranienburg, traduit de l'allemand par Guy Altmann (ed. originale 1945)

Albert Bigielman, J'ai eu 12 ans à Bergen-Belsen, 2008.

Chodzko,Mieczyslaw 2010. Evadé de Treblinka, traduit du polonais par Béatrice Nowak \& Isabelle Choko

Golgevit, Eva. 2010. Ne pleurez pas, mes fils

Goltman, Pierre. 2010. Six mois en enfer

Hirsch, Claude. 2005. Matricule A-16689. Souvenirs de déportation d'un enfant de treize ans - mai 1944 mai 1945 (texte établi en 2002)

Kasza, Élisabeth. 2011. La nomade

Lampel, Gabriel. 2009. K.L.B. 58907 (texte établi avec le concours d'Élisabeth Chombart)

Lichtsztejn-Montard, Sarah. 2011. Chassez les papillons noirs. Récit d'une survivante des camps de la mort nazis

Mitzner, Charles. 2008. Seuls au monde

Rozen-Rechels, Henri. 2008. Je revois... Un enfant juif polonais dans la tourmente nazie 
Skorka-Jacubert, Régine. 2009. Fringale de vie contre usine à mort (entretiens réalisés et mis en forme par Jean Henrion)

Szmidt, Maurice. 2009. Mémoire de déportation (notes originales 1945)

Toros-Marter, Denise. 2008. J'avais seize ans à Pitchipoï

Unger, Julien. 2007. Le sang et l'or. Souvenirs de camps allemands (première édition 1946, Paris : Gallimard)

Woda, Théodore. 2006. Mille jours de la vie d'un déporté qui a eu de la chance

\section{NOTES}

1. Voir Wieviorka (2002) et Joutard (2013). Pour prolonger la réflexion sur les récits de vie sur la Shoah, et le témoignage comme œuvre littéraire, voir Rastier (2005) et dans une bibliographie très importante, lire notamment Jablonka et Wieviorka (2013).

2. On attribue à l'école de Chicago, dans les années 1920, l'origine pratique et conceptuelle des récits de vie (life histories). Depuis lors, la plupart des disciplines en sciences humaines et sociales ont discuté, souvent avec passion, et utilisé les récits de vie. A partir du moment où l'histoire de la Shoah entend se combiner aujourd'hui avec la mémoire de la Shoah, les récits de vies des survivants deviennent essentiels dans le dispositif historiographique (Jablonka et Wieviorka, 2013 : 91-105).

3. Déclinant l'idée de l'indicible de l'horreur, la notion de "faux témoins » a été beaucoup travaillée, bien sûr par les négationnistes mais aussi par certain survivants eux-mêmes, tel Primo Levi, se déclarant inaptes à témoigner de l'horreur et illégitimes, par définition, pour dire une extermination à laquelle ils échappèrent. Pour une discussion de ce point de vue, cf. Rastier (2005 : chap. XII, « l'Art du témoignage », « Mystique du langage et apories de l'indicible »).

4. Centrale dans l'ouvrage d'Agamben (1999), la figure du musulman comme figure emblématique des camps nourrit de manière problématique la thèse de l'impossibilité ontologique du témoignage sur la Shoah. Notre propos est autre : les témoignages - ceux sur la Shoah comme les autres - sont toujours des objets construits a posteriori; ils sont élaboration et réélaboration; pour le témoin, ils sont interprétation de son passé.

5. Symétriquement, on remarque que les pathologies autour des troubles de la mémoire comme Alzheimer affectent en priorité le repérage spatio-temporel des patients.

6. On dira aussi que la perte des repères chronologiques contribue au trauma des déportés.

7. Aussi, certains témoignages renoncent à reconstituer le cadre temporel pour ne proposer, précisément, que des flashs sous la forme de scènes. D'autres récits de vie publiés par la Fondation pour la Mémoire de la Shoah sont accompagnés de notes critiques qui visent notamment à rétablir une chronologie plus cohérente avec le déroulement historique des événements. Si la chronologie dans Si c'est un homme est déjà peu détaillée, Primo Levi y renonce tout à fait dans Les naufragés et les rescapés. Quarante après Auschwitz.

8. Pour finir on se souvient des vers qui ouvrent Si c'est un homme: "Considérez si c'est une femme / Que celle qui a perdu son nom et ses cheveux / Et jusqu'à la force de se souvenir » (Levy 1988).

9. On admettra aussi avec Dosse (1998: 7) que « la mémoire est [...], à l'égal de l'histoire, un mode de sélection dans le passé, une construction intellectuelle ».

10. Nous reprenons ici la démonstration majeure de Guilhaumou sur l'événement historique pour l'appliquer à la mémoire de l'événement : « de l'événementialité, il est donc question ici sur le mode de la donation linguistique : ce qui est donné ne peut être séparé de ce qui est dit, ce qui est dit a une existence pour nous, est donné par le seul fait d'être dit » (Guilhaumou 2006 : 90). 
11. On connait néanmoins les témoignages graphiques et picturaux de survivants tels Issac Celnikier, David Olère, Serge Sumlevic. Mais cela reste marginal par rapport aux centaines de milliers de témoignages langagiers, et nous entrainerait vers une sémiotique de l'image hors de portée de cette contribution. On connait aussi les témoignages documentaires ou filmographiques : ceux-ci sont d'autant plus compliqués à analyser qu'ils accordent eux-mêmes une part déterminante aux témoignages langagiers (narration, interview).

12. Nous reprenons ici librement le terme en référence à la « consolidation de la mémoire » pour les neuro-sciences.

13. Comme la morphologie du terme l'indique, la commémoration relève de l'acte social et moins du souvenir intime.

14. Denise Toros-Marter, J'avais seize ans à Pitchipoï, 2008

15. D'un point de vue méthodologique, on remarquera, en conséquence, que le terme "mère » est au sein du lexique familial celui qui est le moins à droite du graphique.

16. (Cyrulnik 2012a) montre ainsi comment les zones cérébrales activées lors de la convocation d'un souvenir sont très proches des zones activées lors d'une projection imaginaire ou à venir.

17. Iramuteq : www.iramuteq.org.

18. Nous opérons donc une simple compression du texte du corpus en ne gardant que les 300 substantifs sélectionnés mais en respectant l'ordre sur l'axe syntagmatique. Ainsi la phrase « Le maitre lance la balle à son chien » est ramenée à « maitre balle chien ».

19. Sarah Lichtsztejn-Montard, Chassez les papillons noirs. Récit d'une survivante des camps de la mort nazis, Paris, Éditions Le Manuscrit, FMS- Collection Témoignages de la Shoah, 2011

20. Gephi : https://gephi.org/

21. Deux mots sont reliés par un trait s'ils sont co-occurrentts (i.e. s'ils apparaissent ensemble dans le même paragraphe). L'épaisseur des traits rend compte de l'importance de cette cooccurrence, c'est-à-dire de la valeur des cellules du tableau.

22. La principale nuance concerne la fusion des deux classes généalogique (« père », « mère ») et historique (« histoire », «France », « Pologne ») dans la même communauté (en bleue clair) ; fusion guère étonnante puisque la $\mathrm{CDH}$ les avaient reliées fermement; fusion évidemment intéressante pour notre problématique puisque nous cherchons à démontrer que ce vocabulaire partage une dimension co-mémorielle à distance de l'événement proprement vécu.

23. Sarah Lichtsztejn-Montard, Chassez les papillons noirs. Récit d'une survivante des camps de la mort nazis, 2011

24. Mieczyslaw Chodzko, Evadé de Treblinka, 2011

25. Gabriel Lampel, K.L.B. 58907, 2009, Texte établi avec le concours d'Élisabeth Chombart

26. Mieczyslaw Chodzko, Evadé de Treblinka, 2010

\section{ABSTRACTS}

Testimonials of Holocaust survivors are multilayered discursive objects caught between History and memory. We argue that this polarization is embedded in the weft of the narratives in the form of lexical traces that can be untangled quantitatively. To do this, we focus on the most frequent nouns used in a corpus of 16 biographies of survivors from Auschwitz, and use 3 complementary analytical apparatus - correspondence analysis, hierarchical classification and a network-based analysis - to identify the lexical stocks structuring the corpus. Our results confirm 
the polarization of the narratives between a primary mnemonic content and a secondary historical/collective re-presented one.

Entre mémoire et histoire, les récits des témoins de la Shoah sont des objets discursifs riches et complexes. Nous postulons qu'ils portent en eux l'événement traumatique, sous la forme de traces lexicales et d'organisations textuelles sous-jacentes. Les substantifs les plus utilisés sont d'abord extraits d'un corpus de 16 biographies de survivants d'Auschwitz, puis trois traitements de la trame co-occurrenttielle des récits sont proposés grâce à l'Analyse factorielle des Correspondances (AFC), la Classification descendante hiérarchique (CDH) et les réseaux. Au final, nous identifions deux lexiques imbriqués qui structurent les textes : un lexique "primaire » ou mémoriel relevant d'une mémoire intime proche de l'événement et un lexique « secondaire » ou historique relevant d'une mémoire sociale en partie réélaborée.

\section{INDEX}

Keywords: death narrative, discourse analysis, history, holocaust, memory, textual analysis Mots-clés: analyse de données textuelles, analyse du discours, histoire, mémoire, récit de mort, Shoah

\section{AUTHORS}

\section{DAMON MAYAFFRE}

Université de Nice Sophia Antipolis, CNRS

\section{MAHÉ BEN HAMED}

Université de Nice Sophia Antipolis, CNRS 